\title{
INVESTIGACIONES
}

\section{La Autocalificación como Instrumento de Aprendizaje en una Asignatura Universitaria Inversa*}

\author{
Self-assessment as a Learning Instrument in a Flipped University Course
}

\author{
Juan Carlos Luís Pascual ${ }^{a}$,Beatriz Muros Ruiz \\ ${ }^{a}$ Universidad Autónoma de Madrid \\ Correo electrónico: juan.luis@uah.es \\ ${ }^{\mathrm{b}}$ Universidad Autónoma de Madrid \\ Correo electrónico: beatriz.muros@uah.es
}

\section{RESUMEN}

La investigación tiene como objetivo valorar la satisfacción, las ventajas e inconvenientes de la incorporación del aprendizaje inverso dentro de una de propuesta de autocalificación como herramienta de aprendizaje en la asignatura de Enseñanza de la Actividad Física y el Deporte II. Se ha planificado para llevarse a cabo durante el curso 2016-2017 en el grado de Ciencias de la Actividad Física y del Deporte de la Universidad de Alcalá con los estudiantes matriculados en dicha asignatura. El estudio se compone básicamente de un cuestionario que se pasa al terminar la asignatura. Esta indagación forma parte de una práctica sistemática que se repite en cada curso académico donde el docente pone en marcha una intervención específica de evaluación que se valora y que sirve como práctica educativa.

Palabras claves: evaluación formativa, autoevaluación, aprendizaje inverso, enseñanza, universidad.

\section{ABSTRACT}

This research aims to assess the satisfaction, advantages, and disadvantages associated with the incorporation of inverse learning within a proposal of self-assessment as a learning instrument in the course Physical Activity and Sport Teaching II. The study was designed to be carried out during the 2016-2017 academic year in the Physical Activity and Sport Sciences degree program at Alcala University with the students enrolled in the course. The study included a questionnaire that was distributed at the end of the course. This inquiry is part of a systematic practice that is repeated every academic year in which the teacher initiates a specific assessment intervention that is evaluated and that serves as a practice of educational innovation.

Keywords: formative assessment, self-assessment, flipped learning, teaching, university.

Proyecto I+D. Título del Proyecto: Las competencias docentes en la formación inicial del profesorado de educación física. Investigadores principales: Víctor Manuel López Pastor y Antonio Fraile Aranda (Universidad de Valladolid). Centro: Ministerio de Educación. Período 2013-2016. Referencia EDU 2013- 42024-R. Presupuesto: $35000 €$. 


\section{INTRODUCCIÓN}

Esta investigación educativa es un estudio de caso (a nivel micro), que es llevado a cabo por el propio profesor-investigador para analizar la intervención docente realizada en una asignatura universitaria obligatoria de la Universidad de Alcalá durante el curso académico 2016-2017. Se encuadra junto a otros estudios de caso similares, en una investigaciónacción (a nivel macro) realizada por docentes de distintas universidades españolas que se desarrolla en ciclos anuales, coincidiendo con los cursos académicos. Utiliza una experiencia de innovación educativa que implementa una mejora en las competencias propuestas en la asignatura. El docente oferta tres opciones de calificación personal entre las que hay que elegir una: examen final, mixta y autocalificación. En todos los casos, los estudiantes realizan las mismas actividades colaborativas que incluyen actividades de aula, diseño y desarrollo de actividades prácticas, elaboración de un ensayo temático de revisión bibliográfica (al modo y manera de un artículo de revisión), elaboración y defensa de un póster (al modo y manera de la presentación en un congreso).

Las actividades individuales llevadas a cabo por los estudiantes dependen de la modalidad de calificación seleccionada. Por ejemplo, la propuesta de clase invertida se utiliza tanto en la modalidad mixta como en la de autocalificación, para las lecturas previas a los debates en clase, y sirve además como documento de consulta para los estudiantes que opten por el examen final (renunciando a la evaluación continua).

Utilizando la propuesta metodológica de la clase invertida, se informa a los estudiantes sobre el tema a tratar antes de la clase. Todo ello les permite mejorar sus conocimientos sobre dicho tema con el tiempo suficiente para preparar el debate. El docente sigue la consigna siguiente: cuanto mayor conocimiento tenga un alumno sobre un tema, más posibilidades tiene de participar en el aula. Simultáneamente, al docente le sirve de feedback para saber los conocimientos previos y las dificultades que podrían tener los estudiantes en alguno de los apartados de los temas que se utilizan para desarrollar las competencias. Además, ello permite disponer de más tiempo en las sesiones de clase, el que se emplea para dar respuesta a preguntas personales o dar feedback en los trabajos colaborativos y los debates grupales llevados a cabo en el aula.

Las sesiones de clase práctica son más participativas al incorporar explícitamente la evaluación, co-evaluación y autoevaluación. Dicha intervención por parte de los compañeros no condiciona la calificación de las tareas organizadas y desarrolladas por un tercero, aunque sean objeto de evaluación entre todos. Al contrario, es dicha valoración realizada por los propios estudiantes-evaluadores (organizadores, observadores participantes y observadores sistemáticos) la que es objeto de calificación.

En la práctica de innovación implementada se ha seguido un protocolo con la pretensión de hacerla más efectiva: No realizar la organización de la propuesta de innovación con más de 12 grupos colaborativos, para que todos los grupos puedan llevar a cabo un diseño y desarrollo mínimo de tres sesiones prácticas de aplicación de las sesiones teóricas; la asistencia y participación de todas las modalidades de calificación, incluidas la autocalificación y la mixta, para poder asegurar el desarrollo de las actividades colaborativas demandadas; realización de una tutoría previa obligatoria con los grupos pequeños antes de presentar la actividad práctica definitiva; reservar un tiempo de la asignatura para la defensa de la calificación (individualizada). 


\subsection{APRENDIZAJE INVERTIDO}

El aprendizaje inverso (flipped learning) tiene diferentes niveles de aplicación: enseñanza justo a tiempo, enseñanza por compañeros o aprendizaje basado en equipos. El aprendizaje invertido supone una propuesta más activa del aprendizaje llevado a cabo por cada estudiante y también una mejora en la gestión del tiempo. Conlleva poder dedicar más tiempo al trabajo colaborativo de los estudiantes y al aumento del número de los feedback personales implementados por parte del docente en el aula, tal y como plantean (Lee, Lim \& Kim, 2017). Mientras que Ihm, Choy y Roh (2017) destacan la mejora de las habilidades de discusión (reflexión crítica) y la satisfacción de esta propuesta en la clase. Insistiendo en esta misma línea, para Prieto (2017) el modelo de aprendizaje inverso también mejora "los resultados de los aprendizajes de los alumnos" [...] "las interacciones profesoralumno" [...] o "la satisfacción del profesor con el desarrollo de sus alumnos" (p. 70).

El aprendizaje inverso es una propuesta que mejora la interacción del profesorado con sus estudiantes, dentro y fuera del aula, con un trato más personalizado, con una mayor orientación y con la aportación de mayor cantidad de feedback. Entre las características que se explicitan, se pueden destacar las que plantean Tourón y Santiago (2015) al indicar que el aprendizaje inverso permite al docente compartir la información y atender a la diversidad particular de los ritmos de aprendizaje del alumnado. En este sentido, al estudiante se le deja volver a los contenidos de aprendizaje todas las veces que quiera, le interese o necesite. Además, Manrique Arribas (2016) añade la creación de un buen ambiente de trabajo activo y colaborativo que fomentan la creatividad y el pensamiento crítico. Tanto Chocarro de Luis, Santiago y Navaridas (2014) como Tourón y Santiago (2015) consideran que el aprendizaje inverso permite al docente hablar el mismo idioma de las TIC que maneja el estudiante. Recalcando que, por su atractivo para el docente, es un proceso irreversible que no tiene vuelta atrás.

Por otra parte, Osman, Jalal y Azizi (2017) resaltan como ventajas del aprendizaje invertido la mayor flexibilidad de los aprendizajes y la mejora en la regulación de su ritmo de aprendizaje, y, como desventajas, la mayor dificultad y peor preparación de los estudiantes que se descuelgan en el transcurso del proceso a lo largo del curso. Otra de las desventajas, que plantean Camerino y Prieto (2015) es la excesiva carga de trabajo, motivada por la corrección individual previa que tiene que realizar el docente en el caso de grupos muy numerosos.

\subsection{AUTOCALIFICACIÓN}

La relación entre la evaluación y la calificación como herramienta de aprendizaje es revisada desligando la autocalificación de la autoevaluación, dado que lo importante es la mejora de los aprendizajes y no de las calificaciones. La autocalificación no es un proceso automático y se adquiere gradualmente. A este respecto, Sicilia (2010) plantea la falta de costumbre que tiene el alumnado a tener dicho grado de responsabilidad y autonomía, además del peso del credencialismo social y la competitividad del mercado de trabajo.

El uso de la autocalificación sin criterios de evaluación promueve la autorregulación, pero no garantiza aprendizajes profundos, tal y como plantean Panadero y Alonso (2013), al no darse la reflexión crítica. Además, apuntan que la utilización de una rúbrica analítica, 
con criterios de evaluación, ayuda a mejorar los resultados académicos de los estudiantes, especialmente si se asocian a guiones reflexivos de autoevalución (con preguntas que el alumnado tiene que responder y que se utilizan durante el proceso). La aportación de dicha información ${ }^{1}$, desde el principio de curso, es una herramienta fundamental para dicha autorregulación y para el control del proceso por parte del estudiante.

La autocalificación permite identificar y hacer conscientes las fortalezas y las debilidades, orientando las propuestas de mejora, pero requiere mantener un adecuado ambiente de confianza, e incluso de complicidad, entre todos los agentes implicados en el proceso de enseñanza-aprendizaje.

Entre los inconvenientes más destacables de la autocalificación están, para Panadero y Alonso (2013), la búsqueda de concordancia con la calificación del docente (o de los compañeros) frente al ajuste de la autocalificación con el grado de aprendizaje. Mientras que para Luis-Pascual (2015) es la falta de implicación en la presentación y defensa de la calificación, aunque se utilice el portafolio individual con las evidencias que aportan los propios estudiantes. Moraza y Antón (2010) indican que la autoevaluación provoca un desconcierto inicial de los estudiantes y cierta desconfianza que posteriormente se torna en satisfacción y mayor competencia profesional.

El Puzzle Graduado en Luis-Pascual y Tornero Castejón (2011), y más tarde el Contrato Graduado, surgen como una propuesta que mejora la adaptación del estudiante a su propio proceso de aprendizaje. Para Castejón (2009) y Luis-Pascual (2009), las propuestas graduadas tienen como factor común la negociación previa de la calificación con cada estudiante, mediante un acuerdo o un contrato explícito, basado en un compromiso de trabajo individual y opcional, que permite desvincular la calificación de la autoevaluación, mejorando la participación en su propio aprendizaje y en el de sus compañeros. La autocalificación supone un gran paso para la autonomía y responsabilidad de la formación personal (en este caso como futuro profesional docente).

\section{METODOLOGÍA}

La investigación tiene como objetivo valorar la satisfacción, las ventajas e inconvenientes de la incorporación del aprendizaje inverso dentro de una propuesta de autocalificación como herramienta de aprendizaje.

Se plantea una metodología de investigación mixta, tratando de obtener información del mismo grupo de participantes desde dos perspectivas diferentes. Por una parte, desde un punto de vista cualitativo (a nivel micro) como investigación educativa, tal y como plantea Ruiz de Olabuénaga (2012) para la mejora de la propia práctica docente. Por otra parte, desde un punto de vista cuantitativo (a nivel macro) como investigación sobre educación, obteniendo unos datos que forman parte del estudio científico-técnico de la Red Interuniversitaria de Evaluación Formativa y Compartida y planteando ciclos anuales de investigación-acción para la valoración de una práctica de innovación en una de las asignaturas que imparte el docente investigador participante.

En referencia a los indicadores de logro y los criterios de calificación de las competencias seleccionadas. 


\subsection{ANTECEDENTES DE LA INVESTIGACIÓN}

La propuesta de innovación llevada a cabo forma parte del proceso de mejora y perfeccionamiento profesional docente del profesor comenzado hace cuatro años en la asignatura de los estudios de CCAFYDE: Enseñanza de la actividad física y el deporte II. Dicha asignatura es la tercera y última (de las obligatorias) relacionadas con la pedagogía, didáctica, enseñanza y metodología en el Plan de estudios del Grado, pudiéndose completar con otras cuatro optativas.

En el curso académico 2016-2017 se ha mejorado la propuesta sobre autocalificación y clase invertida que comenzó esbozándose en el curso anterior con peticiones voluntarias de las presentaciones o de las lecturas asociadas a cada tema. Mientras que en este curso 2017-2018 se fuerza a la lectura y visionado de los documentos, requiriendo la contestación y cumplimentación de una serie de preguntas cortas, que se entregan antes de la clase. Tal actividad aporta a los estudiantes la información previa necesaria para participar, con mayor conocimiento de causa, en los debates planteados en el aula. En dicho curso, además, se ha conseguido valorar (con anticipación) el grado de comprensión y los apartados de cada tema que requieren más tiempo (con relación a la información complementaria durante su desarrollo en la propia clase), al exigir la respuesta argumentada y reflexiva de 10 preguntas cortas sobre dicho documento (que es enviado previamente). También, se ha incluido una pregunta para que se explicite el tiempo que se ha empleado en dicha tarea por el propio estudiante.

Se mantiene una oferta abierta y personalizada de calificación de cursos anteriores (con tres alternativas), que permite al estudiante adaptar la participación del propio proceso de aprendizaje a sus intereses y circunstancias particulares, tratando de incrementar así su motivación para conseguir aprendizajes más relevantes.

Se depura, además, la rúbrica de calificación de las competencias utilizada por el docente, ofertándose como documento base de negociación de un contrato de aprendizaje individualizado entre las tres opciones posibles de compromiso y calificación: examen final, mixta (contrato graduado) y autocalificación.

La muestra que responde al cuestionario es de 66 estudiantes sobre una población matriculada en la asignatura de 77 alumnos.

\subsection{INSTRUMENTOS DE INVESTIGACIÓN, EVALUACIÓN Y CALIFICACIÓN}

\section{Instrumentos de investigación}

Se utiliza un cuestionario anónimo, validado por la propia Red de Evaluación Formativa y Compartida, para todos y cada uno de los profesores-investigadores (a nivel micro) que forman parte del Proyecto I+D (a nivel macro). Básicamente, el instrumento consiste en una escala Likert con 5 opciones (ver tabla 4), a saber: nada, poco, algo, bastante y mucho.

CUESTIONARIO I: Grado de satisfacción de la propuesta de innovación docente, así como sus ventajas e inconvenientes. Éste se llevó a cabo al final del curso (ver Tabla 4, Tabla 5 y Tabla 6). 
Estudios Pedagógicos XLIV, $\mathrm{N}^{\circ}$ 2: 79-91, 2018

LA AUTOCALIFICACIÓN COMO INSTRUMENTO DE APRENDIZAJE EN UNA ASIGNATURA UNIVERSITARIA INVERSA

Tabla 1. Relación de actividades y criterios de calificación de la opción autocalificación

\begin{tabular}{|c|c|c|}
\hline \multicolumn{2}{|c|}{ Evaluación (opción autocalificación) } & Calificación \\
\hline Actividades de aprendizaje & Instrumento & $\%$ \\
\hline 1. Defensa de la calificación & Rúbrica (opción autocalificación) & $100 \%$ \\
\hline 2. Selección crítica y recogida de evidencias & Portafolio personal (autocalificación) & requisito \\
\hline 3. Elaboración y evaluación de artículo & Carpeta de aprendizaje colaborativo & requisito \\
\hline 4. Visionado de videos & Preguntas cortas & requisito \\
\hline 5. Lecturas críticas temáticas & Preguntas cortas & requisito \\
\hline
\end{tabular}

\section{Instrumentos de evaluación y calificación}

En relación a la metodología y la dinámica de la clase, en este apartado se identifican las actividades e instrumentos de evaluación y calificación con los porcentajes referentes a cada uno de éstos últimos. La primera tabla (ver Tabla 1), se refiere a la opción de autocalificación, la segunda (ver Tabla 2) hace mención a la propuesta de examen final y la tercera (ver Tabla 3) está asociada a la alternativa mixta, a la que se refiere la guía docente por defecto. 72 estudiantes han optado por la autocalificación, 1 por la opción mixta y los 4 restantes no han seleccionado una opción, y han ido a la convocatoria extraordinaria.

Tabla 2. Actividades y criterios de calificación de la opción examen final

\begin{tabular}{|c|c|c|}
\hline \multicolumn{2}{|c|}{ Evaluación (opción examen final) } & Calificación \\
\hline Actividades de aprendizaje & Instrumento & $\%$ \\
\hline 1. Examen final & Preguntas opción múltiple (final) & $100 \%$ \\
\hline 2. Elaboración y evaluación del artículo & Carpeta de aprendizaje colaborativo & requisito \\
\hline 3. Visionado de vídeos & Preguntas cortas & requisito \\
\hline 4. Lecturas críticas temáticas & Preguntas cortas & requisito \\
\hline
\end{tabular}

Tabla 3. Actividades y criterios de calificación de la opción: mixta

\begin{tabular}{|c|l|c|}
\hline \multicolumn{2}{|c|}{ Evaluación (calificación mixta-contrato graduado) } & Calificación \\
\hline Actividades de aprendizaje & Instrumento & $\%$ \\
\hline 1. Examen final & Preguntas opción múltiple (mixta) & $40 \%$ \\
\hline 2. Evaluación de artículos & Lista de control & $30 \%$ \\
\hline 3. Evaluación de presentaciones & Rúbrica & $30 \%$ \\
\hline 4. Elaboración del artículo & Carpeta de aprendizaje colaborativo & requisito \\
\hline 5. Visionado de videos & Preguntas cortas & requisito \\
\hline 6. Lecturas críticas temáticas & Preguntas cortas & requisito \\
\hline
\end{tabular}




\section{RESULTADOS}

En el proceso de análisis de los datos, se explicitan en cada uno de los tres cuestionarios empleados:

\subsection{GRADO DE SATISFACCIÓN, VENTAJAS E INCONVENIENTES DE LA EXPERIENCIA}

Los datos del cuestionario anónimo que se pasa al alumnado al final del curso en referencia al grado de satisfacción (ver Tabla 4), ventajas (ver Tabla 5) e inconvenientes (ver Tabla 6).

Tabla 4. Grado de satisfacción de la experiencia

\begin{tabular}{|c|c|c|c|c|c|c|}
\hline \multirow{2}{*}{ Ítems del cuestionario } & \multicolumn{5}{|c|}{ Poner una " $X$ " en el nivel con un porcentaje más alto en cada ítem } & \multirow{2}{*}{$\begin{array}{l}\text { MED } \\
(1-5)\end{array}$} \\
\hline & Nada & Poco & Algo & Bastante & Mucho & \\
\hline $\begin{array}{l}\text { 1.¿Se ha negociado la utilización } \\
\text { de esta experiencia en la } \\
\text { asignatura al comienzo del } \\
\text { curso? }\end{array}$ & & & & $\mathrm{X}$ & & 4,01 \\
\hline $\begin{array}{l}\text { 2. ¿Crees que esta experiencia te } \\
\text { ha ayudado a adquirir } \\
\text { competencias profesionales? }\end{array}$ & & & $\mathrm{X}$ & & & 3,36 \\
\hline $\begin{array}{l}\text { 3.¿La evaluación que se ha } \\
\text { planteado favorece la adquisición } \\
\text { de las competencias } \\
\text { profesionales? }\end{array}$ & & & $\mathrm{X}$ & & & 3,50 \\
\hline $\begin{array}{l}\text { 4.¿Consideras útil lo aprendido } \\
\text { con esta experiencia? }\end{array}$ & & & $\mathrm{X}$ & & & 3,16 \\
\hline $\begin{array}{l}\text { 5.¿Qué es lo más útil que has } \\
\text { aprendido? } \\
\text { ¿Qué es una experiencia: }\end{array}$ & $\begin{array}{c}\text { 1-Muy } \\
\text { insuficiente }\end{array}$ & 2- Insuficiente & 3-Suficiente & 4- Buenas & $\begin{array}{l}\text { 5- Muy } \\
\text { buenas }\end{array}$ & $\begin{array}{l}\text { MED } \\
(1-5)\end{array}$ \\
\hline $\begin{array}{l}\text { 5.1. innovadora, porque desarro- } \\
\text { lla soluciones nuevas o creativas }\end{array}$ & & & & $\mathrm{X}$ & & 3,6 \\
\hline $\begin{array}{l}\text { 5.2. efectiva, porque } \\
\text { demuestra un impacto } \\
\text { positivo y tangible de } \\
\text { mejora }\end{array}$ & & & $\mathrm{X}$ & & & 3,23 \\
\hline $\begin{array}{l}\text { 5.3. sostenible, porque se } \\
\text { mantiene en el tiempo y puede } \\
\text { producir efectos duraderos }\end{array}$ & & & $\mathrm{X}$ & & & 3,26 \\
\hline $\begin{array}{l}\text { 5.4. replicable, cuando es posible } \\
\text { utilizarla como modelo para } \\
\text { desarrollarla en otros contextos }\end{array}$ & & & & $\mathrm{X}$ & & 3,63 \\
\hline
\end{tabular}




\begin{tabular}{|c|c|c|c|c|c|c|}
\hline & \multicolumn{6}{|c|}{ Poner una "X" en el nivel con un porcentaje más alto en cada ítem } \\
\hline & $\begin{array}{c}\text { 1-Muy } \\
\text { insuficiente. }\end{array}$ & 2-Insuficiente & 3-Suficiente & 4- Buenas & $\begin{array}{l}\text { 5- Muy } \\
\text { buenas }\end{array}$ & $\begin{array}{l}\text { MED } \\
(1-5)\end{array}$ \\
\hline $\begin{array}{l}\text { 6.¿Cómo valoras las ayudas } \\
\text { recibidas por el docente? }\end{array}$ & & & $\mathrm{X}$ & & & 3,13 \\
\hline \multirow{2}{*}{$\begin{array}{l}\text { 7.¿Cómo valoras las ayudas } \\
\text { recibidas por los compañeros? }\end{array}$} & & & $\mathrm{X}$ & & & 3,76 \\
\hline & $\begin{array}{c}\text { 1-Nada } \\
\text { satisfecho }\end{array}$ & $\begin{array}{l}\text { 2-Poco } \\
\text { satisfecho }\end{array}$ & $\begin{array}{l}\text { 3-Medio } \\
\text { Satisfecho }\end{array}$ & $\begin{array}{l}\text { 4- Bastante } \\
\text { satisfecho }\end{array}$ & $\begin{array}{c}\text { 5- Muy } \\
\text { satisfecho }\end{array}$ & $\begin{array}{l}\text { MED } \\
(1-5)\end{array}$ \\
\hline $\begin{array}{l}\text { 8.Señala la satisfacción global en } \\
\text { relación con la experiencia }\end{array}$ & & & $\mathrm{X}$ & & & 2,95 \\
\hline $\begin{array}{l}\text { 9.Señala la satisfacción global en } \\
\text { relación con la evaluación de la } \\
\text { experiencia }\end{array}$ & & & & $\mathrm{X}$ & & 3,43 \\
\hline $\begin{array}{l}\text { 10.¿Cuál es el grado de dificultad } \\
\text { de la experiencia? }\end{array}$ & & & $\mathrm{X}$ & & & 2,95 \\
\hline
\end{tabular}

Con relación al grado de satisfacción (ver Tabla 4) se aprecia que el mayor número de respuestas se encuentra en el grado de satisfacción media, destacando como bastante satisfecho $(\mathrm{M}=4,01)$ la negociación realizada por el docente al principio de curso: alternativas de calificación, rúbrica de calificación y contrato de aprendizaje. En el otro lado, los ítems más bajos $(M=2,95)$ están asociados al grado de satisfacción global con la experiencia y al grado de dificultad de la experiencia.

Tabla 5. Ventajas percibidas por el grupo de estudiantes

\begin{tabular}{|c|c|c|c|c|c|c|c|}
\hline \multirow{2}{*}{$\begin{array}{l}\text { Aspectos que se consideran por parte } \\
\text { del alumnado }\end{array}$} & \multicolumn{5}{|c|}{$\begin{array}{c}\text { Poner una " } \mathrm{X} \text { " en el nivel con un porcentaje } \\
\text { más alto en cada ítem }\end{array}$} & \multirow{2}{*}{$\begin{array}{c}\text { Media } \\
(1-5)\end{array}$} & \multirow[t]{2}{*}{ SD } \\
\hline & Nada & Poco & Algo & Bastante & Mucho & & \\
\hline $\begin{array}{l}\text { 1.Ofrece alternativas a todos los } \\
\text { estudiantes }\end{array}$ & & & & $X$ & & 4,2 & 0,77 \\
\hline $\begin{array}{l}\text { 2.Hay un contrato previo, negociado y } \\
\text { consensuado del sistema de } \\
\text { evaluación }\end{array}$ & & & & & $X$ & 4,6 & 0,70 \\
\hline $\begin{array}{l}\text { 3. Está centrado en el proceso, } \\
\text { importancia del trabajo diario }\end{array}$ & & & & $\mathrm{X}$ & & 4,06 & 0,82 \\
\hline $\begin{array}{l}\text { 4.El estudiante realiza un aprendizaje } \\
\text { activo }\end{array}$ & & & & $X$ & & 3,8 & 0,84 \\
\hline $\begin{array}{l}\text { 5.Se plantea el trabajo en equipo de } \\
\text { forma colaborativa }\end{array}$ & & & & $X$ & & 3,5 & 0,83 \\
\hline \multirow{2}{*}{$\begin{array}{l}\text { 6.El alumno/a está más motivado, } \\
\text { el proceso de aprendizaje es más } \\
\text { motivador }\end{array}$} & & & $\mathrm{X}$ & & & 3,03 & 0,85 \\
\hline & & & & & & & \\
\hline
\end{tabular}




\begin{tabular}{|c|c|c|c|c|}
\hline 7.La calificación es más justa & & $\mathrm{X}$ & 3,65 & 1,02 \\
\hline $\begin{array}{l}\text { 8.Mejora la tutela académica } \\
\text { (seguimiento y ayuda al alumno/a) }\end{array}$ & $\mathrm{X}$ & & 3,09 & 1,06 \\
\hline 9.Permite aprendizajes funcionales & & $\mathrm{X}$ & 3,43 & 0,97 \\
\hline 10.Genera aprendizajes significativos & & $\mathrm{X}$ & 3,61 & 0,88 \\
\hline 11.Se aprende mucho más & $\mathrm{X}$ & & 3,14 & 0,88 \\
\hline $\begin{array}{l}\text { 12.Mejora la calidad de los trabajos } \\
\text { exigidos }\end{array}$ & $\mathrm{X}$ & & 3,22 & 1 \\
\hline $\begin{array}{l}\text { 13.Hay interrelación entre teoría y } \\
\text { práctica }\end{array}$ & & $\mathrm{X}$ & 3,65 & 0,89 \\
\hline $\begin{array}{l}\text { 14.Evalúa todos los aspectos posibles } \\
\text { (en referencia al saber, saber hacer y } \\
\text { saber estar y ser) }\end{array}$ & & $\mathrm{X}$ & 3,86 & 1 \\
\hline $\begin{array}{l}\text { 15.Hay retroalimentación en } \\
\text { documentos y actividades }\end{array}$ & $\mathrm{X}$ & & 3,03 & 1 \\
\hline $\begin{array}{l}\text { 16. Hay posibilidad de corregir errores } \\
\text { en documentos y actividades }\end{array}$ & & $\mathrm{X}$ & 3,49 & 1,16 \\
\hline $\begin{array}{l}\text { 17.Se da un seguimiento más } \\
\text { individualizado }\end{array}$ & $\mathrm{X}$ & & 2,72 & 0,91 \\
\hline 18.Requiere más responsabilidad & & $\mathrm{X}$ & 4,17 & 0,82 \\
\hline
\end{tabular}

Con respecto a las ventajas de la experiencia de innovación implementada por el docente (ver Tabla 5), se aprecia que las valoraciones de los estudiantes se distribuyen entre el ítem algo y el ítem bastante. Destacan, por un lado, la puntuación de mucho $(M=4,6)$ en la negociación del contrato previo y el consenso del sistema de evaluación. Por otro lado, la puntuación de algo $(\mathrm{M}=2,72)$ con relación al seguimiento individualizado llevado a cabo por el docente.

Tabla 6. Inconvenientes percibidos por el grupo de estudiantes

\begin{tabular}{|c|c|c|c|c|c|c|c|}
\hline \multirow{2}{*}{$\begin{array}{l}\text { Aspectos que se consideran por } \\
\text { parte del alumnado }\end{array}$} & \multicolumn{5}{|c|}{$\begin{array}{l}\text { Poner una "X" en el nivel con un porcentaje } \\
\text { más alto en cada ítem. }\end{array}$} & \multirow{2}{*}{ Media (1-5) } & \multirow{2}{*}{$\mathrm{SD}$} \\
\hline & Nada & Poco & Algo & Bastante & Mucho & & \\
\hline $\begin{array}{l}\text { 1.Exige una asistencia } \\
\text { obligatoria y activa }\end{array}$ & & & & $\mathrm{X}$ & & 4,14 & 1,06 \\
\hline $\begin{array}{l}\text { 2. Tiene una dinámica de } \\
\text { trabajo poco conocida, falta de } \\
\text { hábito }\end{array}$ & & & & $\mathrm{X}$ & & 3,95 & 0,87 \\
\hline 3.Exige continuidad & & & & $\mathrm{X}$ & & 4,11 & 0,85 \\
\hline $\begin{array}{l}\text { 4.Hay que comprenderlo } \\
\text { previamente }\end{array}$ & & & & $\mathrm{x}$ & & 3,94 & 0,97 \\
\hline
\end{tabular}




\begin{tabular}{|c|c|c|c|c|c|c|}
\hline 5.Exige un mayor esfuerzo & & & $\mathrm{X}$ & & 3,84 & 0,92 \\
\hline $\begin{array}{l}\text { 6.Existe dificultad para trabajar } \\
\text { en grupo }\end{array}$ & & $\mathrm{X}$ & & & 3,26 & 1,06 \\
\hline $\begin{array}{l}\text { 7.Se puede acumular mucho } \\
\text { trabajo al final }\end{array}$ & & $\mathrm{X}$ & & & 3,2 & 1 \\
\hline $\begin{array}{l}\text { 8.Existe una desproporción } \\
\text { trabajo/créditos }\end{array}$ & $\mathrm{x}$ & & & & 2,66 & 1,16 \\
\hline $\begin{array}{l}\text { 9.El proceso de calificación es } \\
\text { más complejo y, a veces, poco } \\
\text { claro }\end{array}$ & & & $\mathrm{X}$ & & 3,49 & 1,03 \\
\hline $\begin{array}{l}\text { 10.Genera inseguridad e } \\
\text { incertidumbre, dudas sobre que } \\
\text { hay que realizar }\end{array}$ & & & & $\mathrm{X}$ & 4,26 & 0,94 \\
\hline $\begin{array}{l}\text { 11.Es injusto frente a otros } \\
\text { procesos de evaluación }\end{array}$ & $\mathrm{X}$ & & & & 2,61 & 1,33 \\
\hline $\begin{array}{l}\text { 12.Las correcciones han sido } \\
\text { poco claras }\end{array}$ & & $\mathrm{X}$ & & & 3,18 & 1,16 \\
\hline $\begin{array}{l}\text { 13.La valoración del trabajo es } \\
\text { subjetiva }\end{array}$ & & & $\mathrm{X}$ & & 3,39 & 0,92 \\
\hline $\begin{array}{l}\text { 14.Exige participar en mi } \\
\text { propia evaluación } \\
\text { (autoevaluarse) }\end{array}$ & & & & $\mathrm{X}$ & 4,69 & 0,73 \\
\hline
\end{tabular}

En relación con los inconvenientes (ver Tabla 6) hay una mayor dispersión de las puntuaciones, comparada con el grado de satisfacción y con las ventajas. Se puede encontrar entre las más altas la de mucho $(\mathrm{M}=4,26)$, el ítem asociado a las dudas, inseguridades e incertidumbres de la propuesta llevada a cabo por el docente y también $(M=4,69)$ el ítem ligado a la exigencia de que el estudiante participe en su propia evaluación (autoevaluación). Entre las más bajas la de algo $(M=2,66)$, relacionada con el grado de desproporcionalidad entre trabajo y créditos adscritos a la asignatura; y también la de algo $(\mathrm{M}=2,61)$ vinculada con la injusticia frente a otros procesos de evaluación.

\section{DISCUSIÓN}

La valorización realizada por los estudiantes es satisfactoria respecto a la propuesta de intervención realizada en la asignatura. En el análisis de la escala Likert se observa siempre la respuesta media a partir de algo satisfactorio, marcando como bastante satisfactorio la evaluación global de la propuesta de innovación. A este respecto, se pueden encontrar referencias que reflejan un aumento de la calidad de las reflexiones y la satisfacción de los estudiantes (Lee et al., 2017) cuando se implementa el aprendizaje inverso en el aula, incluso combinando aprendizaje inverso y autoevaluación. También se constata la satisfacción del alumnado en Koo, Demps, Farris, Bowman y Panahi (2016) y en Wang (2017). 
Respecto a las ventajas de la propuesta, la percepción del grupo de alumnos se sitúa entre la media (algo) y la media-alta (bastante). Destacando y reconociendo de forma expresa la negociación (aumento de la participación, responsabilidad y toma de decisiones) llevada a cabo con los estudiantes en relación con la modalidad de calificación y los compromisos (contrato) de aprendizaje, al puntuarse como muy alta (mucho). En este mismo sentido, se recoge de una manera similar que la evaluación formativa mejora el compromiso, la autorregulación y el seguimiento que realiza el estudiante del proceso de enseñanza-aprendizaje en Hortigüela et al. (2017).

Como área de mejora surge el seguimiento personal llevado a cabo por el docente. Esto supone que en la introducción progresiva del flipped-learning en la asignatura, en un primer momento se solicitó la lectura y visionado de materiales con los que se iba a trabajar en clase; en un segundo momento se encargó la respuesta a una serie de preguntas cortas reflexivas sobre dichos materiales, contestando a algunos estudiantes al azar; y en un tercer momento (como propuesta de futuro) se vincula con dar un feedback a todo el alumnado, antes de la clase, para animar los debates. Camerino y Prieto (2015) también señalan como ventaja la "posibilidad de trabajar individualmente sobre las correcciones colectivas realizadas en clase" (p. 278). No obstante, se puede combinar la metodología flipped-learning con sesiones magistrales sin perder efectividad (Borchardt \& Bozer, 2017) frente a la utilización exclusiva de clases magistrales.

Con relación a los inconvenientes, surgen y se explicitan las preocupaciones de los estudiantes en relación con la incertidumbre de lo que puede pasar, al no estar todo tan cerrado por parte del docente; la inseguridad que provoca el no poner límites a la partición de los compañeros (co-evaluación); y la propia valoración personal (autoevaluación) o las dudas generadas por la responsabilidad en la elección del sistema de calificación.

Aunque está en el bloque de inconvenientes, se valora bien (como adecuada) la carga de trabajo realizada y su correspondencia en tiempos y créditos ECTS. En esta línea, Ihm, Choy y Roh (2017, p. 97) señalan que con el flipped-learning se consigue "maximizar la efectividad de sus procesos de aprendizaje".

Con relación a la calificación, específicamente si es justa o no, se percibe por el alumnado como satisfactoria, implementándose el conocimiento previo de los criterios de calificación negociados por el grupo-aula y la valoración, de su consecución y estado intermedio, a la mitad de la asignatura. Además, con la obligación de aportar evidencias de los procesos de enseñanza-aprendizaje propios y de los compañeros en los que se ha participado.

El proceso planteado en la propuesta de innovación se ha refinado al incorporar de una manera más sistematizada los instrumentos de evaluación que permiten guiar mejor las observaciones que llevan a cabo los estudiantes. Se ha mejorado la implicación y la calidad de dicha participación, discriminando niveles de participación en los debates de clase (opinión, descripción, comparación, interpretación y teorización). Se han incluido herramientas del aula virtual que marcan un momento de entrega, facilitando los registros y la posibilidad de dar feedback. Se ha incluido una valoración personal explícita (en mitad del proceso) a modo de evidencia, sobre el grado de consecución de las competencias que aparecen en la rúbrica. Se ha regularizado el proceso de preguntas que realiza el docente sobre la defensa de la calificación llevada a cabo y las evidencias aportadas en el portafolio por el estudiante. Se han incorporado otras fórmulas para tratar de clarificar la práctica y reducir la incertidumbre que supone la toma de decisiones, entre ellas un documento 
sobre las preguntas frecuentes que tratan de informar a los estudiantes sobre la práctica de innovación llevada a cabo, las opciones de calificación y los requisitos que suponen cada una de ellas.

Así, se proponen como soluciones a los problemas encontrados: devolución de la información sobre las tareas individuales previas reclamadas a los estudiantes (aprendizaje inverso); aumento del número de feedback personales a todos y cada uno de los alumnos antes de las sesiones de clase, sobre la documentación entregada previamente en el campus virtual, a dichas clases; clarificación de la comprensión, asegurando que los estudiantes hayan entendido y no tengan dudas sobre la oferta de calificación que realiza el docente.

Las propuestas de mejora para el próximo curso académico giran en torno a la personalización del feedback realizado a los estudiantes antes de la clase sobre los trabajos previos solicitados (especialmente respuestas a las cuestiones planteadas por el docente a los vídeos y lecturas entregados).

\section{CONCLUSIONES}

El grado general de satisfacción que perciben los estudiantes respecto a la propuesta de innovación llevada a cabo por el docente es positivo y, por tanto, pueden considerarse válidas las propuestas metodológicas llevadas a cabo por el docente, de aprendizaje inverso y autocalificación asociadas, para la mayor parte del alumnado.

También es satisfactoria la percepción de la calificación finalmente obtenida por los estudiantes, gracias al conocimiento previo de los criterios de calificación, su revisión explícita por parte de los estudiantes a la mitad del curso y la necesidad de aportar evidencias sobre los propios aprendizajes llevados a cabo, y sobre la colaboración en los aprendizajes de los compañeros del grupo pequeño y del grupo-aula.

Respecto a las ventajas de la propuesta de intervención, destaca y se reconoce de forma expresa la negociación llevada a cabo con los estudiantes en relación con la elección de la modalidad de calificación al contrato de aprendizaje. Se valora bien, como adecuada y pertinente, la carga de trabajo empleada y su correspondencia en cuanto a tiempo asignado y créditos ECTS.

En relación con los inconvenientes, surgen y se mantienen las preocupaciones clásicas de los estudiantes frente a las metodologías más activas: la incertidumbre inicial de lo que puede pasar, al estar abiertos a la negociación los criterios de calificación de la asignatura; la inseguridad que provoca el no saber dónde están los límites de la co-evaluación de los compañeros; y la propia autocalificación.

\section{REFERENCIAS BIBLIOGRÁFICAS}

Borchardt, J., \& Bozer, A.H. (2017). Psychology course redesign: an interactive approach to learning in a micro-flipped classroom. Smart Learning Environments, 4(10), 1-9.

Camerino, O., \& Prieto, A. (2015). Flipped Classroom y la evaluación continuada con TIC. En N. González-Fernández, I. Salcines y E. García-Ruiz (Coords.), Tendencias emergentes en evaluación formativa y compartida en docencia: el papel de las nuevas tecnologías (pp. 271284). Santander: Universidad de Cantabria. 
Castejón, F.J. (2009). Escala de calificación graduado y elección por el alumnado al principio de la asignatura. En V. López-Pastor (Coord.), Evaluación Formativa y Compartida en Educación Superior. Propuestas, técnicas, instrumentos y experiencias (pp. 150-154). Madrid: Narcea.

Chocarro de Luis, E., Santiago, R., \& Navaridas, F. (2014). Experiencias docentes basadas en el aprendizaje inverso. En A. Mendieta Ramírez (Coord.), Visiones docentes en las aulas de hoy (pp. 85-104). Madrid: ACCI.

Hortigüela, D., Fernández del Río, J., Castejón, F.J., \& Pérez-Pueyo, A. (2017). Formative assessment, work regulation, organization, engagement, tracking and attendance in Spanish Universities. Revista Electrónica Interuniversitaria de Formación del Profesorado, 20(3), 49-63.

Ihm, J., Choi, H., \& Roh, S. (2017). Flipped-learning course design and evaluation through student self-assessment in a predental science class. Korean Journal Medical Education, 29(2), 93-100.

Koo, C.L., Demps, E.L., Farris, C., Bowman, J., \& Panahi, L. (2016). Impact of Flipped Classroom Design of Student Performance and Perceptions in a Pharmacotherapy Course. American Journal of Pharmaceutical Education, 80(2), 1-9.

Lee, J., Lim, C., \& Kim, H. (2017). Development of an instructional design model for flipped leaning in higher education. Educational Technology Research and Development, 65(2), 427-453.

Luis-Pascual, J.C. (2009). Experiencia 2 Sistema de contrato graduado. En V. López-Pastor (Coord.), Evaluación Formativa y Compartida en Educación Superior. Propuestas, técnicas, instrumentos y experiencias (pp. 155-159). Madrid: Narcea.

Luis-Pascual, J.C. (2015). Inconvenientes de la autocalificación de las competencias en la educación superior. Estudio de caso en la Universidad de Alcalá. En M. Jabonero, M. Martín, A. Martínez y J.L. Bizelli (Eds.), Miradas diversas de la educación en Iberoamérica (pp. 553-570). Bucaramanga: Universidad Autónoma de Bucaramanga / Fundación Santillana.

Luis-Pascual, J.C., \& Tornero Castejón, A. (2011). El Puzzle Graduado a debate. En C. Canabal, M.D. García-Campos, M. I. Gegúndez y C. Viejo (Coords.), La creación de espacios comunes de aprendizaje (pp. 191-209). Alcalá de Henares: Universidad de Alcalá.

Manrique Arribas, J.C. (2016). El Flipped Classroom un modelo pedagógico ideal para aplicar la evaluación formativa y compartida. Trabajo presentado en I Jornadas de Buenas Prácticas en Evaluación Formativa y Compartida en Docencia Universitaria, León, Universidad de León.

Moraza, J.I., \& Antón, A. (2010). La evaluación compartida: estudio comparativo entre la autoevaluación y autocalificación del alumnado y la evaluación y calificación del profesor. International Journal of Developmental and Educational Psychology, 3(1), 97-104.

Osman, A., Jalal, S.R., \& Azizi, S. (2017). Flipped learning: should it replace didactic learning? Advances in Medical Education and Practice, 8, 707-708.

Panadero-Calderón, E., \& Alonso-Tapia, J. (2013). Revisión sobre autoevaluación educativa: evidencia empírica de su implementación a través de la autocalificación sin criterios de evaluación, rúbricas y guiones. Revista de investigación en educación, 11(2), 172-197.

Prieto, A. (2017). Flipped learning: Aplicar el modelo de aprendizaje inverso. Madrid: Narcea.

Ruiz de Olabuénaga, I. (2012). Metodología de la investigación cualitativa (5a ed.). Bilbao: Universidad de Deusto.

Sicilia, A. (2010). Libertad, autonomía y libertad en el proceso de autoevaluación y autocalificación. En A. Sicilia (Ed.), La evaluación y calificación en la Universidad: relatos autobiográficos durante la búsqueda de alternativas (pp. 1-14). Barcelona: Hipatia.

Tourón, J., \& Santiago, R. (2015). El modelo Flipped Learning y el desarrollo del talento en la escuela. Revista de Educación, 368, 196-231.

Wang, F.H. (2017). An exploration of online behavior engagement and achievement in flipped classroom supported by learning management system. Computers \& Education, 114, 79-91. 
